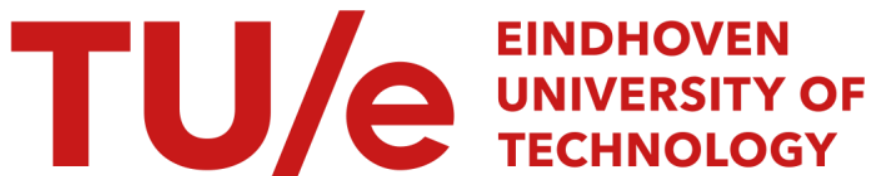

\section{Multi-component droplet growth. II. A theoretical model}

\section{Citation for published version (APA):}

Peeters, P., Pieterse, G., \& Dongen, van, M. E. H. (2004). Multi-component droplet growth. II. A theoretical model. Physics of Fluids, 16(7), 2575-2586. https://doi.org/10.1063/1.1751192

DOI:

10.1063/1.1751192

Document status and date:

Published: 01/01/2004

\section{Document Version:}

Publisher's PDF, also known as Version of Record (includes final page, issue and volume numbers)

\section{Please check the document version of this publication:}

- A submitted manuscript is the version of the article upon submission and before peer-review. There can be important differences between the submitted version and the official published version of record. People interested in the research are advised to contact the author for the final version of the publication, or visit the $\mathrm{DOI}$ to the publisher's website.

- The final author version and the galley proof are versions of the publication after peer review.

- The final published version features the final layout of the paper including the volume, issue and page numbers.

Link to publication

\section{General rights}

Copyright and moral rights for the publications made accessible in the public portal are retained by the authors and/or other copyright owners and it is a condition of accessing publications that users recognise and abide by the legal requirements associated with these rights.

- Users may download and print one copy of any publication from the public portal for the purpose of private study or research.

- You may not further distribute the material or use it for any profit-making activity or commercial gain

- You may freely distribute the URL identifying the publication in the public portal.

If the publication is distributed under the terms of Article 25fa of the Dutch Copyright Act, indicated by the "Taverne" license above, please follow below link for the End User Agreement:

www.tue.nl/taverne

Take down policy

If you believe that this document breaches copyright please contact us at:

openaccess@tue.nl

providing details and we will investigate your claim. 


\title{
Multi-component droplet growth. II. A theoretical model
}

\author{
P. Peeters, G. Pieterse, and M. E. H. van Dongen \\ Eindhoven University of Technology, Department of Applied Physics, P.O. Box 513, \\ $5600 \mathrm{MB}$ Eindhoven, The Netherlands
}

(Received 24 July 2003; accepted 10 March 2004; published online 4 June 2004)

\begin{abstract}
A droplet growth model is formulated that describes the growth of homogeneous multi-component droplets suspended in a dilute gas-vapor mixture. The droplet is suspended in a real carrier gas, in which the vapors are diluted. The model is applicable for all Knudsen numbers. It is shown that the model can easily be extended to describe the growth of layered droplets. The model calculations are compared to the results of droplet growth experiments of supersaturated water and/or supersaturated $n$-nonane in methane. The model performs well for all the cases, except for the case when supersaturated $n$-nonane vapor is added to supersaturated water vapor in methane gas. In the latter case it is proposed that the discrepancies originate from the incomplete wetting of $n$-nonane on liquid water at the given conditions. (C) 2004 American Institute of Physics.
\end{abstract}

[DOI: $10.1063 / 1.1751192]$

\section{INTRODUCTION}

In the previous paper experimental droplet growth results of water and $n$-nonane in methane are presented. ${ }^{1}$ There, it is shown that supersaturated $n$-nonane can grow onto water droplets, resulting in a growing droplet consisting of a water core and a $n$-nonane coating. In this paper we will present a theoretical model that can describe the growth of such coated droplets, suspended in a dilute gas-vapor mixture.

The growth of droplets (or, similarly, evaporation of droplets) is determined by the rate of mass and energy transfer from and towards the droplet. At steady state, these fluxes are constant. In most cases, the growth of the droplets can be assumed to be quasi-steady. This means that the boundary conditions which define the growth rate vary slowly compared to the time the system needs to adapt to the new conditions. In this way the whole growth process can be divided into small time intervals. In each interval the growth is assumed to be steady, at the given (averaged) conditions of the time interval. The choice of appropriate expressions describing the mass and energy fluxes depends on the Knudsen number $\mathrm{Kn}$, which is a length-scale parameter. Here, it is defined as the ratio of a characteristic mean free path of the molecules $\lambda$, which will be defined later, to the diameter of the droplet. For very large Knudsen numbers the transfer of heat and mass is dominated by the impingement rate of molecules onto the surface of the droplet, and is accordingly described by gas kinetics. ${ }^{2,3}$ This is the so-called HertzKnudsen regime. For very small Knudsen numbers the transfer of mass and energy is accurately described by continuum relations.

Droplets that start to grow after the nucleation stage (the formation of the smallest possible stable droplets) will generally grow from the regime of very large Knudsen numbers to the regime of very small Knudsen numbers. To describe this process, a model is needed that incorporates the transition regime between the Knudsen regime and the continuum regime. For the description of droplet growth at intermediate Knudsen numbers there exist several approaches. Gyarmathy suggested an interpolating fitting function between the two limiting cases. ${ }^{4}$ In this way, growth rates of single component droplets can accurately be described. Fuchs and Sutugin gave correction factors with which the continuum fluxes need to be multiplied in order to describe droplet growth in the transition regime. ${ }^{5}$ In their approach the Knudsen number for mass transfer differs from the Knudsen number for heat transfer. The correction factors of heat and mass transfer are functions of their respective Knudsen numbers. Vesala and Kulmala and Kulmala et al. used these corrections to describe the growth of uniform multi-component droplets. ${ }^{6,7} \mathrm{~A}$ different approach to the description of transitional droplet growth is given by the flux-matching method. ${ }^{8}$ According to this model the droplet is surrounded by a Knudsen layer. The layer has a thickness of the order of the mean free path of the molecules, and mass and heat transfer are governed by gas kinetics within this region. Outside the Knudsen layer the transfer rates are described by continuum relations. In steady state the mass and energy fluxes in both regions are equal and constant, giving rise to a set of equations describing the growth for all values of the Knudsen number. This method was first applied for mass fluxes only by Fuchs, and later extended by Fukuta and Walter to include the energy flux.9,10

Here, we will adopt the flux-matching model. First, we will use it to describe the growth of multi-component homogeneous droplets. Next, we will extend the model in order to describe the growth of a layered droplet, i.e., a droplet consisting of a homogeneous core and a different homogeneous shell. The results of the formulated droplet growth models will be compared to the experimental results presented in the first paper. ${ }^{1}$

\section{HOMOGENEOUS DROPLET MODEL}

In Fig. 1 the growing droplet is shown schematically. The droplet is assumed to be spherical with radius $r_{d}$, and to 


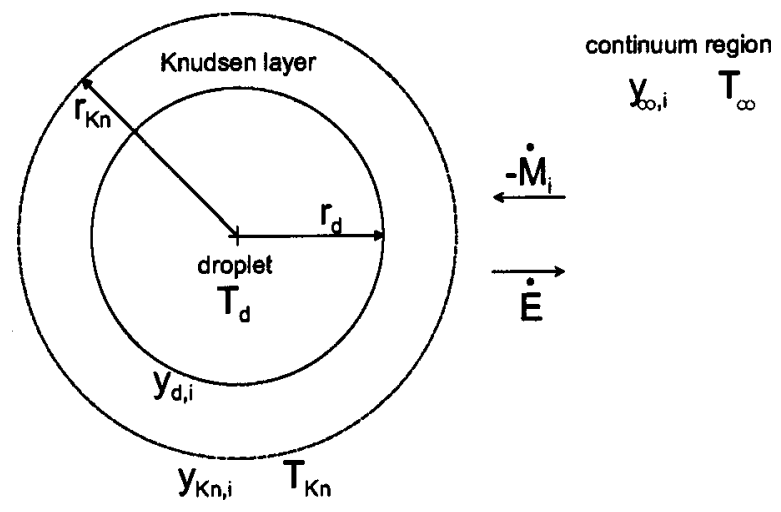

FIG. 1. Schematic view of a growing droplet.

have a uniform temperature $T_{d}$. The Knudsen layer surrounds the droplet, and has a radius $r_{\mathrm{Kn}}$. The total molar flux $\dot{M}$ and energy flux $\dot{E}$ are defined positive when directed away from the droplet. The velocity distribution at $r_{\mathrm{Kn}}$ differs from the equilibrium Maxwell velocity distribution due to the mass and energy fluxes. Young assumed a Grad velocity distribution at $r_{\mathrm{Kn}}$, describing single-component droplet growth in an ideal inert carrier gas. ${ }^{11,12}$ This model was made applicable to droplet growth in high pressure inert carrier gases by Peeters et al. and by Luijten, by incorporating realgas effects. ${ }^{13,14}$ We will now further modify this model to include both the effect of dissolution of the carrier gas into the droplet and the condensation of more than one vapor component.

\section{A. Continuum region}

For the determination of the fluxes in the continuum region we will use conservation of mass and energy. For a spherically symmetric steady flow problem the conservation of mass is given by the condition that the total molar flux is invariant,

$$
\frac{\mathrm{d} \dot{M}}{\mathrm{~d} r}=\frac{\mathrm{d}}{\mathrm{d} r}\left(r^{2} \rho^{G} u\right)=0,
$$

where $u$ is the mean molar (radial) velocity of the gas, and $\rho^{G}$ is the molar density of the gas phase. In case viscous effects are neglected, the conservation of energy is given by

$$
\frac{\mathrm{d} \dot{E}}{\mathrm{~d} r}=\frac{\mathrm{d}}{\mathrm{d} r}\left(r^{2} \rho^{G} u\left(h^{G}+\frac{M_{\text {mix }} u^{2}}{2}\right)+r^{2} \dot{q}\right)=0,
$$

where $h^{G}$ is the molar enthalpy of the gas, $M_{\text {mix }}$ is the molar mass of the gas, and $\dot{q}$ is the heat flux per unit area. The pressure is assumed to be uniform throughout the entire gas phase, which is valid for small Mach numbers $(\mathrm{Ma}=|u / c|$ $\ll 1$, with $c$ the speed of sound).

\section{Molar fluxes}

The (radius-independent) total molar flux in the continuum region is given by the integral of Eq. (1). The total molar density is given by

$$
\rho^{G}=\rho_{g}^{G}+\sum_{j} \rho_{j}^{G}
$$

where $\rho_{g}^{G}$ is the carrier gas density and $\rho_{j}^{G}$ is the density of vapor component $j$. The velocity of each component is equal to the sum of the bulk velocity $u$ and its diffusive velocity $v$. Furthermore, by definition one has

$$
\rho_{g}^{G} v_{g}+\sum_{j} \rho_{j}^{G} v_{j}=0
$$

Combining Eqs. (3) and (4) with Eq. (1) and integrating gives

$$
\begin{aligned}
\dot{M} & =4 \pi r^{2} \rho_{g}^{G}\left(u+v_{g}\right)+4 \pi r^{2} \sum_{j} \rho_{j}^{G}\left(u+v_{j}\right) \\
& =\dot{M}_{g}+4 \pi r^{2} \sum_{j} \rho_{j}^{G}\left(u+v_{j}\right),
\end{aligned}
$$

where $\dot{M}$ is the total molar flux. When considering dilute vapors the diffusive velocities can be approximated using Fick's law of diffusion in the form

$$
\rho_{j}^{G} v_{j}=-\rho^{G} D_{j}^{G} \frac{\mathrm{d}}{\mathrm{d} r} \frac{\rho_{j}^{G}}{\rho^{G}},
$$

where $D_{j}^{G}$ is the diffusion coefficient of component $j$ in the gas phase. Substituting Fick's law of diffusion into Eq. (5) and introducing the molar vapor fraction $y_{j}=\rho_{j}^{G} / \rho^{G}$ one obtains

$$
\dot{M}\left(1-\sum_{j} y_{j}\right)=\dot{M}_{g}-4 \pi r^{2} \rho^{G} \sum_{j} D_{j}^{G} \frac{\mathrm{d}}{\mathrm{d} r} y_{j} .
$$

For small vapor fractions one has $\left(1-\Sigma_{j} y_{j}\right) \approx 1$, and the equation can easily be integrated from $\left(r_{\mathrm{Kn}} ; y_{\mathrm{Kn}, j}\right)$ to $(r$ $\left.=\infty ; y_{\infty, j}\right)$. The result is

$$
\dot{M}=\dot{M}_{g}-4 \pi r_{\mathrm{Kn}} \rho^{G} \sum_{j} D_{j}^{G}\left(y_{\infty, j}-y_{\mathrm{Kn}, j}\right) \equiv \dot{M}_{g}+\sum_{j} \dot{M}_{j} .
$$

To derive an expression for the total molar flux of the carrier gas molecules $\dot{M}_{g}$ we assume that the gas is constantly in equilibrium with the droplet. The total flux of carrier gas molecules is then determined by the growth rate of the droplet times the equilibrium gas fraction in the droplet, which can be written as

$$
\dot{M}_{g}=-\frac{\mathrm{d}}{\mathrm{d} t}\left(\frac{4}{3} \pi r_{d}^{3} x_{g} \rho^{L}\right),
$$

where $x_{g}$ is the molar carrier gas fraction in the liquid, and $\rho^{L}$ is the molar density of the liquid. For inert carrier gases the equilibrium liquid molar fraction is negligible, and the flux of carrier gas molecules also becomes zero.

\section{Energy flux}

The (radius-independent) total energy flux in the continuum region is given by the integral of Eq. (2), giving

$$
\dot{E}=4 \pi r^{2} \rho^{G} u\left(h^{G}+\frac{M_{\mathrm{mix}} u^{2}}{2}\right)+4 \pi r^{2} \dot{q} .
$$

The heat flux per unit area is given by 


$$
\dot{q}=-k \frac{\mathrm{d} T}{\mathrm{~d} r}+h_{g} \rho_{g}^{G} v_{g}+\sum_{j} h_{j} \rho_{j}^{G} v_{j},
$$

where $k$ is the heat conductivity of the gas mixture, and $h_{i}$ is the partial molar enthalpy. As can be seen from Eq. (11) the heat flux is a combination of heat conduction and the transport of enthalpy by mass diffusion. The total enthalpy of the gas is given by

$$
h^{G}=\frac{\rho_{g}^{G}}{\rho^{G}} h_{g}^{G}+\sum_{j} \frac{\rho_{j}^{G}}{\rho^{G}} h_{j}^{G} .
$$

Equations (11) and (12) are substituted into Eq. (10). Because the mean mass velocity is small, we neglect the term due to the kinetic energy of the mean flow in Eq. (10). The result then is

$$
\dot{E}=\dot{M}_{g} h_{g}^{G}+\sum_{j} \dot{M}_{j} h_{j}^{G}-4 \pi r^{2} k \frac{\mathrm{d} T}{\mathrm{~d} r},
$$

where Eq. (5) was used for the molar fluxes. When the enthalpy $h$ is approximated by $c_{p} T$, where $c_{p}$ is the molar heat capacity, this equation can be integrated from $\left(r_{\mathrm{Kn}} ; T_{\mathrm{Kn}}\right)$ to $\left(r=\infty ; T_{\infty}\right)$, yielding

$$
\begin{gathered}
\ln \left(\frac{\dot{E}-T_{\mathrm{Kn}}\left(c_{p, g} \dot{M}_{g}+\sum_{j} c_{p, j} \dot{M}_{j}\right)}{\dot{E}-T_{\infty}\left(c_{p, g} \dot{M}_{g}+\Sigma_{j} c_{p, j} \dot{M}_{j}\right)}\right) \\
=\frac{-\left(c_{p, g} \dot{M}_{g}+\Sigma_{j} c_{p, j} \dot{M}_{j}\right)}{4 \pi r_{\mathrm{Kn}} k} .
\end{gathered}
$$

In the continuum region the energy transfer is dominated by conduction. Therefore, the term on the right-hand side of Eq. (14) is small. Taking the exponent of this equation, and then the second order Taylor expansion of the remaining exponential, results in

$$
\begin{aligned}
\dot{E}= & \frac{1}{2}\left(T_{\mathrm{Kn}}+T_{\infty}\right)\left(c_{p, g} \dot{M}_{g}+\sum_{j} c_{p, j} \dot{M}_{j}\right) \\
& +4 \pi r_{\mathrm{Kn}} k\left(T_{\mathrm{Kn}}-T_{\infty}\right) .
\end{aligned}
$$

This is the final result for the total energy flux in the continuum region.

\section{B. Knudsen layer}

In the Knudsen layer, surrounding the droplet, mass and energy transport are determined by gas kinetics. It is assumed that all the molecules that hit the droplet surface fully thermally accommodate, or, equivalently, all thermal accommodation coefficients are assumed to be equal to one. For thermal accommodation coefficients smaller than one the removal of latent heat from the droplet is less effective, and the initial growth rate of the droplet will be smaller. In the continuum regime (i.e., small Knudsen numbers) the influence of the accommodation coefficients on the droplet growth is negligible. Here, all the droplets that leave the droplet surface are assumed to have a Maxwell velocity distribution at temperature $T_{d}$ :

$$
f_{i}^{+}=\left(\frac{M_{i}}{2 \pi R T_{d}}\right)^{3 / 2} \exp \left(-\frac{M_{i}\left(\xi_{r i}^{2}+\xi_{\theta i}^{2}+\xi_{\phi i}^{2}\right)}{2 R T_{d}}\right)
$$

where $\xi_{r i}, \xi_{\theta i}$, and $\xi_{\phi i}$ are the molecular velocities of component $i$ in the $r, \theta$, and $\phi$ directions, and $R$ denotes the universal gas constant. The molecules coming from the Knudsen interface are not in equilibrium. The velocity distribution at the Knudsen interface should correctly describe the net molar and energy fluxes. This is done by the Grad velocity distribution, which in this case is given by ${ }^{12,15}$

$$
\begin{aligned}
f_{i}^{-}= & \left(\frac{M_{i}}{2 \pi R T_{\mathrm{Kn}}}\right)^{3 / 2}\left(1-\frac{\dot{q}_{i}\left(\xi_{r i}-u\right) M_{i}^{2}}{\rho_{i} R^{2} T_{\mathrm{Kn}}^{2}}\right. \\
& \times\left(1-\frac{M_{i}\left(\left(\xi_{r i}-u\right)^{2}+\xi_{\theta i}^{2}+\xi_{\phi i}^{2}\right)}{5 R T_{\mathrm{Kn}}}\right) \\
& \left.+\frac{v_{i}\left(\xi_{r i}-u\right) M_{i}}{R T_{\mathrm{Kn}}}\left(\frac{7}{2}-\frac{M_{i}\left(\left(\xi_{i}-u\right)^{2}+\xi_{\theta i}^{2}+\xi_{\phi i}^{2}\right)}{2 R T_{\mathrm{Kn}}}\right)\right) \\
& \times \exp \left(-\frac{M_{i}\left(\left(\xi_{r i}-u\right)^{2}+\xi_{\theta i}^{2}+\xi_{\phi i}^{2}\right)}{2 R T_{\mathrm{Kn}}}\right)
\end{aligned}
$$

where $q_{i}$ is the partial heat flux per unit area. Because the Knudsen layer has a thickness of the order of the mean free path of the molecules, all the molecules that hit the droplet surface are assumed to have this Grad velocity distribution. The velocity distributions will be used in the derivation of the molar and energy fluxes in the Knudsen layer.

\section{Molar fluxes}

The total molar flux of component $i$ in the Knudsen boundary layer can be expressed as

$$
\begin{aligned}
\dot{M}_{i} & =\alpha_{e v, i} \dot{M}_{i}^{+}-\left(1-\alpha_{c o n, i}\right) \dot{M}_{i}^{-}+\dot{M}_{i}^{-} \\
& =\alpha_{e v, i} \dot{M}_{i}^{+}+\alpha_{c o n, i} \dot{M}_{i}^{-}
\end{aligned}
$$

where $\alpha_{e v}$ and $\alpha_{c o n}$ are the probabilities that molecules escape from or stick to the droplet surface. The total molar flux of evaporating molecules of species $i$ is given by

$$
\begin{aligned}
\dot{M}_{i}^{+} & =4 \pi r_{d}^{2} \int_{0}^{\infty} \int_{-\infty}^{\infty} \int_{-\infty}^{\infty} \rho_{d, i}^{G} \xi_{r i} f_{i}^{+} \mathrm{d} \xi_{r i} \mathrm{~d} \xi_{\theta i} \mathrm{~d} \xi_{\phi i} \\
& =4 \pi r_{d}^{2} \frac{y_{d, i} p}{Z} \frac{1}{\sqrt{2 \pi M_{i} R T_{d}}},
\end{aligned}
$$

where $Z$ is the compressibility factor of the gas phase. Likewise, the total molar flux of incoming molecules of species $i$ is given by 


$$
\begin{aligned}
\dot{M}_{i}^{-}= & 4 \pi r_{d}^{2} \int_{-\infty}^{0} \int_{-\infty}^{\infty} \int_{-\infty}^{\infty} \rho_{\mathrm{Kn}, i}^{G} \xi_{r i} f_{i}^{-} \mathrm{d} \xi_{r i} \mathrm{~d} \xi_{\theta i} \mathrm{~d} \xi_{\phi i} \\
\approx & -4 \pi r_{d}^{2} \frac{y_{\mathrm{Kn}, i} p}{Z} \frac{1}{\sqrt{2 \pi M_{i} R T_{\mathrm{Kn}}}} \\
& \times\left(1-\frac{\left(u+v_{i}\right) \sqrt{\pi M_{i}}}{\sqrt{2 R T_{\mathrm{Kn}}}}\right) \\
= & -4 \pi r_{d}^{2} \frac{y_{\mathrm{Kn}, i} p}{Z} \frac{1}{\sqrt{2 \pi M_{i} R T_{\mathrm{Kn}}}}+\frac{r_{d}^{2}}{2 r_{\mathrm{Kn}}^{2}} \dot{M}_{i} .
\end{aligned}
$$

Substitution of the molar fuxes given by Eqs. (19) and (20) into Eq. (18) results in

$$
\begin{aligned}
\dot{M}_{i}\left(1-\alpha_{c o n, i} \frac{r_{d}^{2}}{2 r_{\mathrm{Kn}}^{2}}\right)= & 4 \pi r_{d}^{2} \frac{p}{Z \sqrt{2 \pi M_{i} R}}\left(\frac{\alpha_{e v, i} y_{d, i}}{\sqrt{T_{d}}}\right. \\
& \left.-\frac{\alpha_{c o n, i} y_{\mathrm{Kn}, i}}{\sqrt{T_{\mathrm{Kn}}}}\right) .
\end{aligned}
$$

For very large Knudsen numbers the ratio $r_{d} / r_{\mathrm{Kn}} \ll 1$, and the formula of Hertz-Knudsen is retrieved. ${ }^{2,3}$ The total molar flux of the carrier gas $\dot{M}_{g}$ can again be given by Eq. (9).

\section{Energy flux}

For the calculation of the energy flux in the Knudsen boundary layer it is assumed that the translational kinetic energy of the molecules is uncorrelated to their rotational and vibrational energy. ${ }^{11}$ In that case the total energy flux is given by

$$
\begin{aligned}
\dot{E}= & \sum_{i}\left\{\left(\dot{M}_{i}-\dot{M}_{i}^{-}\right)\left(c_{p, i}-\frac{R}{2}\right) T_{d}\right\} \\
& +\sum_{i}\left\{\dot{E}_{i}^{-}+\dot{M}_{i}^{-}\left(c_{p, i}-\frac{5 R}{2}\right) T_{\mathrm{Kn}}\right\} .
\end{aligned}
$$

This equation contains two summands on the right-hand side. These are in consecutive order: the energy of the outgoing molecules and the energy of the incoming molecules. Note that the sum is over all components, including the carrier gas. The term $\dot{E}_{i}^{-}$represents the kinetic translational energy of the incoming molecules of component $i$. It is shown in the Appendix that it can be approximated by

$$
\begin{aligned}
\dot{E}_{i}^{-}= & -4 \pi r_{d}^{2} \frac{y_{\mathrm{Kn}, i} p}{Z} \frac{2 R T_{\mathrm{Kn}}}{\sqrt{2 \pi M_{i} R T_{\mathrm{Kn}}}} \\
& +\frac{r_{d}^{2}}{2 r_{\mathrm{Kn}}^{2}}\left(\frac{5}{2} R T_{\mathrm{Kn}} \dot{M}_{i}+\dot{Q}_{\mathrm{Kn}, c i}\right),
\end{aligned}
$$

where $\dot{Q}_{c i}$ is the total energy transfer due to conduction of component $i$. When Eqs. (20) and (23) are substituted into Eq. (22) the final expression for the total energy flux in the Knudsen layer is obtained, which reads

$$
\begin{aligned}
\dot{E}= & 4 \pi r_{d}^{2}\left(T_{d}-T_{i}\right) \sum_{i} \frac{y_{\mathrm{Kn}, i} p}{Z} \frac{c_{p, i}-R / 2}{\sqrt{2 \pi M_{i} R T_{\mathrm{Kn}}}} \\
& +\left(1-\frac{r_{d}^{2}}{2 r_{\mathrm{Kn}}^{2}}\right) T_{d} \sum_{i} \dot{M}_{i}\left(c_{p, i}-\frac{R}{2}\right) \\
& +\frac{r_{d}^{2}}{2 r_{\mathrm{Kn}}^{2}}\left(\dot{Q}_{\mathrm{Kn}, c}+T_{\mathrm{Kn}} \sum_{i} \dot{M}_{i} c_{p, i}\right) .
\end{aligned}
$$

Here, the total heat flux by conduction $\dot{Q}_{\mathrm{Kn}, c}$ is given by

$$
\dot{Q}_{\mathrm{Kn}, c}=\sum_{i} \dot{Q}_{\mathrm{Kn}, c i}=4 \pi r_{\mathrm{Kn}} k\left(T_{\mathrm{Kn}}-T_{\infty}\right) .
$$

\section{Knudsen layer thickness}

To define the boundary of the Knudsen layer $r_{\mathrm{Kn}}$ Young suggested the following relation: ${ }^{11}$

$r_{\mathrm{Kn}}=r_{d}+\beta \lambda$,

where $\lambda$ is the mean free path of the molecules. The corresponding Knudsen number is defined as

$$
\mathrm{Kn}=\frac{\lambda}{2 r_{d}} .
$$

The fluxes are weakly dependent on the choice of the free parameter $\beta$. As a best fit value Young suggests $\beta=0.75$, which we will adopt here. ${ }^{11}$ The value of the mean free path of the molecules $\lambda$ is taken equal to the average distance a molecule has to travel before its velocity is uncorrelated to its initial velocity. This distance depends on the masses of the interacting molecules. For diluted vapors in a carrier gas the dominant molecular interactions are gas-gas (energy transfer) and vapor-gas (mass transfer) interactions. This results in $j+1$ different values for the mean free path, when $j$ is the number of condensing vapor components. However, it was shown by Peeters et al. that for a diluted vapor a single mean free path, calculated from the gas-gas interactions, suffices, as long as $0.43<M_{v} / M_{g}<86 .{ }^{13}$ It can easily be checked that this holds for the mixtures under investigation (water and $n$-nonane in methane), and many other mixtures. Therefore, the mean free path is given by

$$
\lambda=\lambda_{g-g}=\frac{1}{\rho_{g}^{G} d_{g}^{2} N_{A} \pi \sqrt{2}},
$$

where $d_{g}$ is the collision diameter of the carrier gas molecules.

\section{Complete set of equations}

In order to define a complete set of equations, the unknowns have to be defined. These are the molar fluxes $\dot{M}_{j}$ of the $j$ condensing vapor components, the energy flux $\dot{E}$, the temperature $T_{\mathrm{Kn}}$ and molar fractions $y_{\mathrm{Kn}, j}$ of the gas at the Knudsen interface $r_{\mathrm{Kn}}$, and the temperature $T_{d}$ of the droplet. The droplet is assumed to be in quasi-equilibrium with the gas phase. The vapor fractions at the surface of the droplet are determined by the partial saturated vapor pressures of the droplet. These depend on the molar fractions of the vapor 
components in the droplet, rendering another $j-1$ unknowns $\left(\sum x_{j}+x_{g}=1\right)$. This amounts up to $3 j+2$ unknowns, where $j$ is the number of condensing vapor components. In the previous section we have derived $2 j+2$ equations for the $\dot{M}_{j}$ molar fluxes and the energy flux $\dot{E}$. Another $j$ equations can be obtained by introducing the droplet growth rate $\mathrm{d} r_{d} / \mathrm{d} t$, increasing the number of unknowns to $3 j+3$. The $j$ equations are obtained by applying conservation of mass to the droplet, rendering

$$
\dot{M}_{j}=-\frac{\mathrm{d}}{\mathrm{d} t}\left(\frac{4}{3} \pi r_{d}^{3} x_{j} \rho^{L}\right)
$$

Finally, the set is completed by coupling the molar flux of all the components to the energy flux. This can easily be done, since the energy flux originates from the latent heat of condensation of all the vapor components. When the heat release due to the dissolution of the carrier gas into the droplet is neglected one can write

$$
\begin{aligned}
\dot{E} & =\frac{\mathrm{d}}{\mathrm{d} t}\left(\sum_{j} \frac{4}{3} \pi r_{d}^{3} x_{j} \rho^{L} h_{j}^{L}\right) \\
& =\sum_{j} \dot{M}_{j} h_{j}^{L}+\sum_{j} \frac{4}{3} \pi r_{d}^{3} x_{j} \rho^{L} \frac{\mathrm{d} h_{j}^{L}}{\mathrm{~d} t} .
\end{aligned}
$$

When considering (quasi) steady growth, the droplet temperature is constant and the second summand on the righthand side equals zero. The equation above can then be rewritten to give

$$
\begin{aligned}
\dot{E} & =-\sum_{j} \dot{M}_{j}\left(h_{d, j}^{G}-h_{j}^{L}\right)+\sum_{j} \dot{M}_{j} h_{d, j}^{G} \\
& =-\sum_{j} \dot{M}_{j}\left(L_{j}-h_{d, j}^{G}\right),
\end{aligned}
$$

where $L_{j}$ is the latent heat of condensation of vapor component $j$.

\section{LAYERED DROPLET MODEL}

In this section the growth rate of a droplet which consists of two liquids is described. The droplet is assumed to have a layered structure. As a first approximation we assume that liquid (2) covers the spherical core with a layer of uniform thickness. The core consists of liquid (1). The layered droplet is shown schematically in Fig. 2. The core has a radius $r_{d c}$ and the layer has an outer radius $r_{d l}$. The energy flux within the droplet is neglected, and the pressure and temperature are assumed to be uniform. The molar and energy fluxes outside the droplet are described similarly as for the homogeneous droplet. The main difference with the homogeneous droplet is the occurrence of a molar flux inside the droplet through the liquid layer. We will limit the study to cases in which the main components of the liquid core are diluted in the liquid layer.

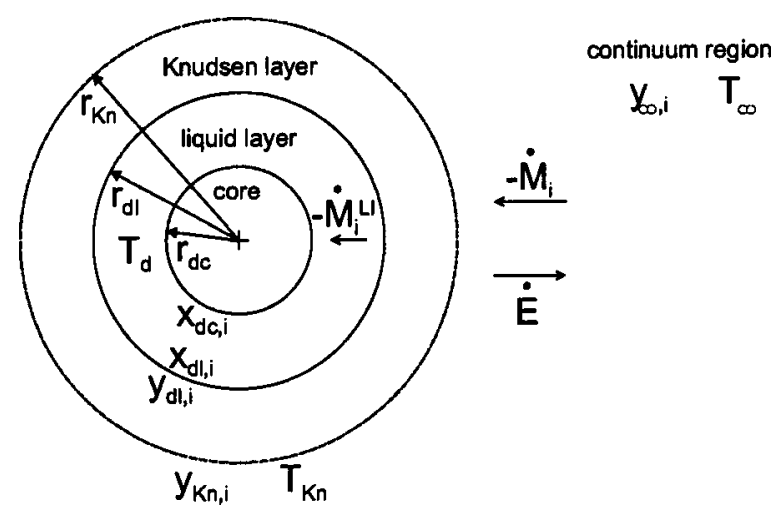

FIG. 2. Schematic view of the growth of a layered droplet.

\section{A. Molar fluxes in liquid layer}

The molar fluxes through the liquid layer are again controlled by diffusion, as was the case in the continuum region in the gas phase. The molar flux of vapor component $k$ through the liquid layer is given by

$$
\dot{M}_{k}^{L l}=4 \pi \frac{r_{d c} r_{d l}}{r_{d c}-r_{d l}} \rho^{L l} D_{k}^{L l}\left(x_{r_{d l}, k}^{L l}-x_{r_{d c}, k}^{L l}\right) .
$$

The vapor components $k$ are components which are diluted in the liquid layer, and which are the main components of the liquid core. The $m$ main (abundant) components of the liquid layer are assumed to be in constant equilibrium with the liquid core. Therefore, the molar flux of the $m$ components in the liquid layer is given by

$$
\dot{M}_{m}^{L l}=-\frac{\mathrm{d}}{\mathrm{d} t}\left(\frac{4}{3} \pi r_{d c}^{3} x_{m}^{L c} \rho^{L c}\right),
$$

similar to Eq. (9). The sum of the vapor components $k$ and $m$ is equal to the total number of condensing vapor components $j$. When the carrier gas molecules enter the droplet, their molar flux is now given by

$$
\dot{M}_{g}=-\frac{\mathrm{d}}{\mathrm{d} t}\left(\frac{4}{3} \pi r_{d c}^{3} x_{g}^{L c} \rho^{L c}+\frac{4}{3} \pi\left(r_{d l}^{3}-r_{d c}^{3}\right) x_{g}^{L l} \rho^{L l}\right),
$$

in which the first term on the right-hand side denotes the molar gas flux through the liquid layer.

\section{B. Complete set of equations}

In the layered droplet model we have $j$ unknown molar vapor fluxes $\dot{M}_{j}$ in the gas phase, $k$ unknown molar fluxes $\dot{M}_{k}^{L l}$ in the liquid layer, and a total of $3 j+k-2$ unknown molar fractions $y_{\mathrm{Kn}, j}, y_{r_{d l}, j}, x_{r_{d l}, j}^{L l}$, and $x_{k}^{L c}$. The term -2 originates from the fact that the sum of all the fractions in the liquid layer and in the liquid core are in each case equal to $\left(1-x_{g}\right)$. Furthermore, we have the unknown temperatures $T_{\mathrm{Kn}}$ and $T_{d}$, the unknown growth rates of the inner core $\mathrm{d} r_{d c} / \mathrm{d} t$ and outer layer $\mathrm{d} r_{d l} / \mathrm{d} t$, and the unknown energy flux $\dot{E}$, rendering a total of $4 j+2 k+3$ unknowns. The molar fractions in the liquid layer at the liquid core are the equilibrium fractions at the given conditions of the liquid layer. 
As before there are $2 j+2$ equations for the molar and energy fluxes in the gas phase, and now another $k$ equations for the molar fluxes in the liquid layer. By applying conservation of mass to the droplet core,

$$
\dot{M}_{k}^{L l}=-\frac{\mathrm{d}}{\mathrm{d} t}\left(\frac{4}{3} \pi r_{d c}^{3} x_{k}^{L c} \rho^{L c}\right),
$$

another $k$ equations are obtained, setting the total number of equations equal to $2 j+2 k+2$. At the surface of the droplet there is continuity of chemical potential for each component. This is also known as local equilibrium between the gas phase and the liquid phase. It results in $j$ equations that relate the molar fraction in the liquid layer at the surface of the droplet $x_{r_{d l}, j}^{L l}$ to the molar fraction in the gas phase at the surface of the droplet $y_{r_{d l}, j}$. Application of conservation of mass of component $j$ in the liquid layer, results in

$$
\dot{M}_{j}^{L l}-\dot{M}_{j}=\frac{\mathrm{d}}{\mathrm{d} t} \int_{r_{d c}}^{r_{d l}} x_{j}^{L l}(r) \rho^{L l} 4 \pi r^{2} \mathrm{~d} r .
$$

The derivation of the radius-dependent fraction of component $j$ in the liquid layer is given in Appendix B. The total number of equations is now equal to $4 j+2 k+2$. The equation that makes the set complete is again obtained from the coupling of the molar flux to the energy flux. The release of heat due to the dissolution of components into the inner core, and the dissolution of gas into the droplet is neglected. If it is then assumed that for all the vapor components the latent heat of condensation is released as soon as they are part of the droplet, Eq. (31) can again be used to describe the coupling between the molar flux and the energy flux. Effectively this means that the release of heat due to the dissolution of the components of the liquid core into the liquid layer is equal to their latent heat of condensation. Then, no additional heat is released when these components diffuse through the liquid layer and reach the inner core.

\section{MIXTURES OF METHANE, $n$-NONANE, AND/OR WATER}

The model calculations are performed using a FORTRAN code in which the system of equations is solved for the each time step (set by the experiment). The system of equations is solved using the standard NAG subroutine C05NCF. After each time step the droplet size is updated and the process is repeated again. In the following the application of the growth model to mixtures of methane, $n$-nonane, and/or water will be discussed, in order to compare the model calculations to the experimental results. ${ }^{1}$ To obtain values for the diffusion coefficient, the Fuller correlation is multiplied by the ratio of the experimental to the calculated value of the diffusion coefficient, which is given by the previous paper. ${ }^{1}$ In this way, small temperature and pressure variations are still accounted for, while the mean value is equal to the experimentally obtained value. In the experiments we do not have a single growing droplet, but a cloud of equisized growing droplets. For all the cases, these droplets are at least 50 times the droplet radius apart. Therefore, the droplets in the cloud can be considered to grow independently. ${ }^{4,16}$ The pressure is re- corded during the experiment, and is almost constant during the growth of the droplets. The temperature and compressibility factor are calculated from the pressure signal, using an equation of state. ${ }^{1,17}$ In the experiment binary mixtures of supersaturated $n$-nonane or water in methane, and ternary mixtures of supersaturated $n$-nonane and water in methane are used. In Sec. IV A the growth calculations for the binary mixtures will be discussed, followed by the growth calculations for the ternary mixtures in Sec. IV B.

\section{A. Binary mixtures}

For the experiments with the binary mixtures there is only one vapor fraction. The initial vapor fraction is obtained from the experiment. As the droplets grow, this vapor fraction is slowly depleted. Therefore, the vapor fraction is a function of time, which can be expressed as

$$
\begin{aligned}
y_{\infty, v}(t)= & y_{\infty, v}\left(t_{0}\right)-n_{d}\left(t_{0}\right) \frac{Z_{\infty}\left(t_{0}\right) R T_{\infty}\left(t_{0}\right)}{p\left(t_{0}\right)} \\
& \times\left(\frac{4}{3} \pi r_{d}^{3}\left(t_{0}\right) x_{v}\left(t_{0}\right) \rho^{L}\left(t_{0}\right)-\int_{t_{0}}^{t} \dot{M}_{v} \mathrm{~d} t\right),
\end{aligned}
$$

where $n_{d}$ is the droplet number density. The initial radius $r_{d}\left(t_{0}\right)$ depends on the supersaturation ratio $S_{v}\left(=y_{v} / y_{v}^{e q}\right)$ of the vapor at which the nucleation of the droplet took place. Muitjens showed that a cluster of molecules can be considered a stable droplet when they contain twice the number of molecules of the critical cluster. ${ }^{18}$ The smallest radius of the droplet is therefore given by

$$
r_{d}\left(t_{0}\right)=2^{1 / 3} \frac{2 \sigma}{x_{v} \rho^{L} R T \ln S_{v}} .
$$

The vapor fraction at the surface of the droplet is obtained using the enhancement factor $f e$, calculated from the CPA equation of state. ${ }^{19-21}$ This results in

$$
y_{r_{d}, v}(t)=f e p_{s a t, v} \exp \left(\frac{2 \sigma}{x_{v} \rho^{L} R T r_{d}}\right),
$$

where the exponential term corrects for the curvature of the droplet. At time $t_{n}$ the system of equations is solved. Then the droplet radius is adjusted according to

$$
r_{d, t_{n+1}}=r_{d, t_{n}}+\left(t_{n+1}-t_{n}\right)\left[\frac{\mathrm{d} r_{d}}{\mathrm{~d} t}\right]_{t=t_{n}},
$$

and the procedure is repeated again. All the physical properties that are directly related to the droplet (like its density) are calculated at the temperature of the droplet $T_{d}$ (for each time step). All the other physical properties (like the thermal conductivity) are calculated at an intermediate temperature $T_{m}$. According to Hubbard et al. a one-third rule is appropriate: ${ }^{22}$

$$
T_{m}=\frac{1}{3}\left(2 T_{d}+T_{\infty}\right) .
$$

In Figs. 3-6 different examples are shown of typical growth curves of either $n$-nonane in methane or water in methane, at 11 bar and $242 \mathrm{~K}$ or 44 bar and $247 \mathrm{~K}$. The agreement is as expected, since the diffusion coefficients 


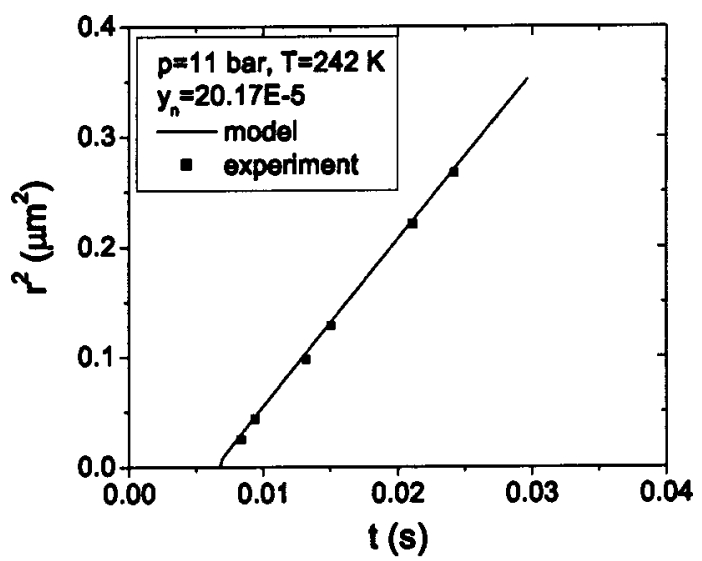

FIG. 3. Growth curve of an $n$-nonane droplet in suspended methane.

were fitted to the experimental results. The growth curves intersect the time axis at about $6.6 \mathrm{~ms}$, which is the time at which the nucleation pulse occurs. ${ }^{1}$ After about $30 \mathrm{~ms}$ the shock wave, which has reflected from the end wall of the low pressure section, reaches the end wall of the high pressure section. Then, temperature and pressure increase again, causing the droplets to evaporate. The decrease in the slope of the growth curve of water in methane at 11 bar and $242 \mathrm{~K}$ (Fig. 5 ) is caused by the depletion of the vapor. The growth curves of $n$-nonane in methane show a small jump at the initial state, which is due to experimental time discretization. The dissolution of methane into the water droplets is in all cases quite small. However, the solubility of methane into liquid $n$-nonane at 44 bar and $247 \mathrm{~K}$ is about $25 \mathrm{~mol} \%$. In order to show the influence of the methane solubility on the growth rate of $n$-nonane droplets, the theoretical growth curve when ignoring the presence of methane is also shown in Fig. 4, for the condition of $44 \mathrm{bar}$ and $247 \mathrm{~K}$. The growth curves with and without methane solubility differ little. This can be explained by the fact that the dissolution of methane into liquid $n$-nonane has little influence on the liquid volume. Or, equivalently, the partial liquid $n$-nonane density in methane/ $n$-nonane mixtures is approximately equal to the pure liquid $n$-nonane density.

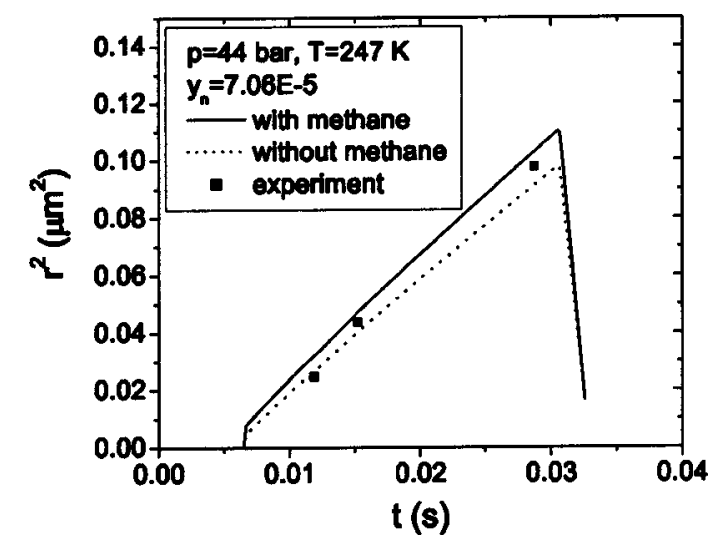

FIG. 4. Growth curve of an $n$-nonane droplet in suspended methane.

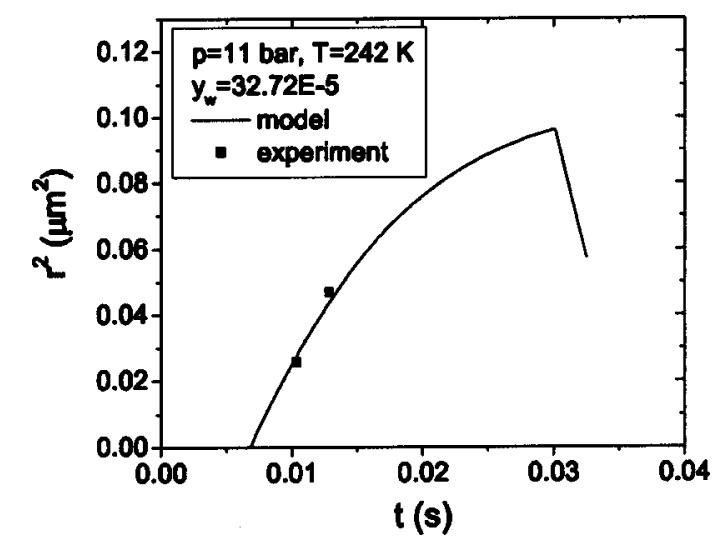

FIG. 5. Growth curve of a water droplet suspended in methane.

\section{B. Ternary mixtures}

For the droplet growth in the ternary mixtures we can distinguish two cases. First we will consider the case in which the $n$-nonane is the dominant nucleating component $\left(J_{n}>J_{w}\right)$. To a very good approximation, the initial cloud of droplets can then be considered to exist of $n$-nonane droplets only. As discussed, it is assumed that the water will not grow onto the $n$-nonane droplets. ${ }^{1}$ The solubility of water into the liquid $n$-nonane is incorporated via ${ }^{19}$

$$
y_{r_{d l}, w}=x_{w}^{L_{n}} \frac{K_{w, n m}(p, T)}{x_{w}^{L n, e q}} \frac{p_{s a t, w}}{p}
$$

where $y_{r_{d l}, w}$ is taken equal to the supersaturated water vapor fraction. The factor $K_{w, n m}$ depends on pressure and temperature, and has been determined using the CPA equation of state which was fitted to experimental phase equilibrium data. ${ }^{19}$ The solubility of methane into the liquid $n$-nonane/ water mixture is taken equal to the solubility of methane into pure liquid $n$-nonane, which is a good approximation due to the very small water fractions in the ternary liquid. For the same reason, the density of the ternary liquid is approximated by the density of liquid $n$-nonane/methane mixtures, at the same conditions. Two examples of the growth calculations are shown in Figs. 7 and 8. The agreement between the experimental results and the model calculations is again very

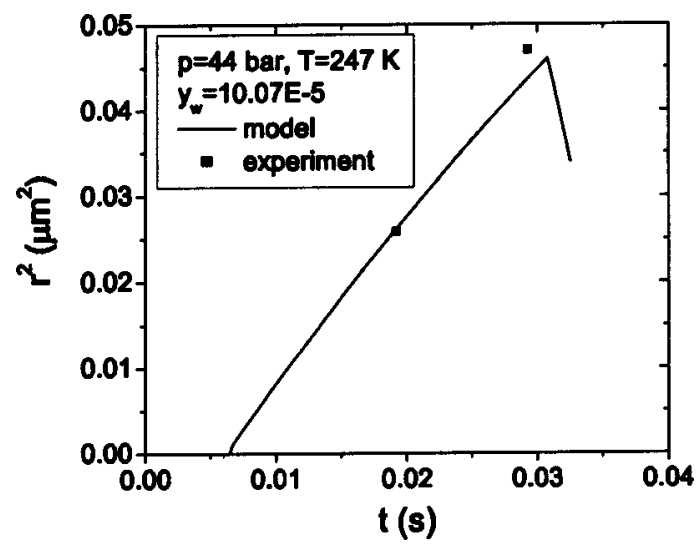

FIG. 6. Growth curve of a water droplet suspended in methane. 


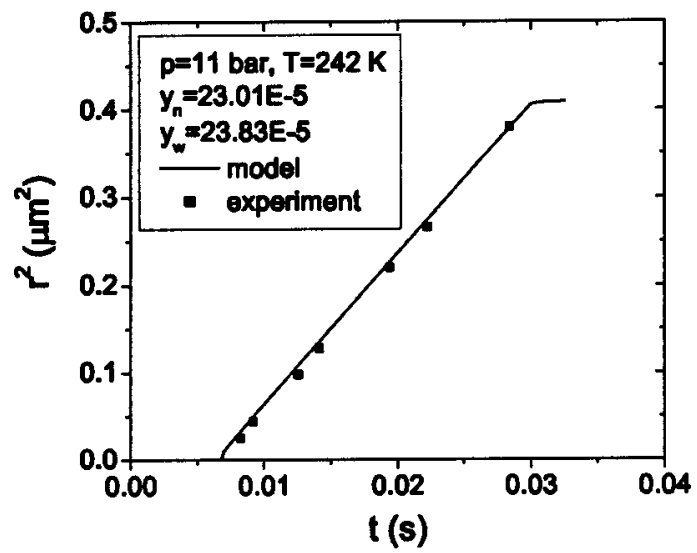

FIG. 7. Growth curve of an $n$-nonane droplet suspended in a mixture of supersaturated water vapor, $n$-nonane vapor, and methane.

good. This indicates that water indeed does not contribute to the growth of the $n$-nonane droplets (the solubility of water into liquid $n$-nonane is negligible).

Now we will consider the case in which water is the dominant supersaturated vapor fraction. The initial cloud of droplets then almost completely consists of water droplets. As discussed in the previous paper, the supersaturated $n$-nonane will then nucleate onto the water droplet, as soon as it has reached a certain minimum size. ${ }^{1}$ We then assume that the droplet will grow further, having an inner core consisting mainly of water and a surrounding liquid layer, which mainly consists of $n$-nonane. This growth mechanism can easily be simulated by only allowing for the dissolution of $n$-nonane into the water droplet, until its radius is equal to the critical radius of the $n$-nonane at the given supersaturation ratio $S_{n}$. Then the $n$-nonane is allowed to grow onto the water droplet. The $n$-nonane vapor fraction at the surface of the droplet is now determined by

$$
y_{r_{d l}, n}=x_{r_{d l}, n}^{L n} \frac{f e_{n m}}{x_{n m}^{e q}} \frac{p_{s a t, n}}{p} \exp \left(\frac{2 \sigma}{x_{n m}^{e q} \rho^{L n} R T r_{d}}\right),
$$

which is an approximation based on the continuity of the chemical potential at the phase boundary. ${ }^{19}$ The exponential term is again included to correct for the curvature of the

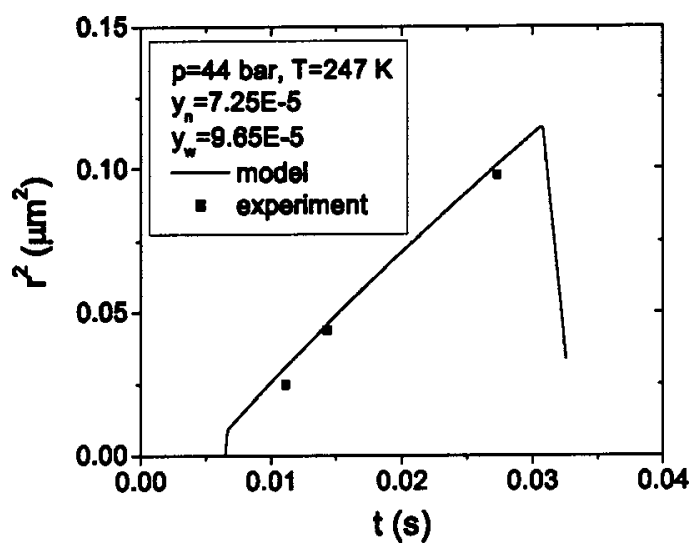

FIG. 8. Growth curve of an $n$-nonane droplet suspended in a mixture of supersaturated water vapor, $n$-nonane vapor, and methane.

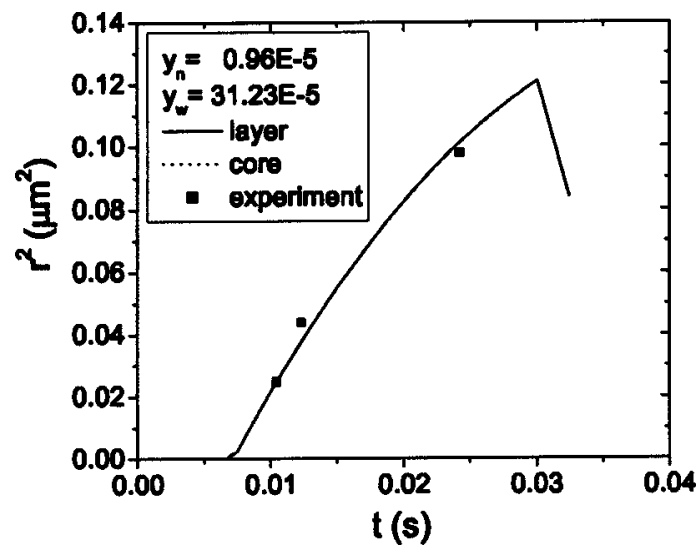

FIG. 9. Growth curve of a water droplet suspended in a mixture of supersaturated water vapor, $n$-nonane vapor, and methane.

droplet. The approximation for the continuity of the chemical potential of the water component is in this case given by

$$
y_{r_{d l}, w}=x_{r_{d l}, w}^{L n} K_{w, n m} \frac{p_{s a t, w}}{p} \exp \left(\frac{2 \sigma}{x_{n m}^{e q} \rho^{L n} R T r_{d}}\right) .
$$

Prior to the heterogeneous nucleation of the $n$-nonane onto the water droplet, the growth can be described as binary, where the mass flux of the $n$-nonane can be approximated by its equilibrium concentration in the liquid water droplet, rendering

$$
\dot{M}_{n}=-\frac{\mathrm{d} r_{d}}{\mathrm{~d} t}\left(\frac{4}{3} \pi r_{d}^{3} x_{n}^{L w} \rho^{L w}\right) .
$$

The vapor depletion and the initial radius of the droplet are calculated in a similar way as was done for the growth in the binary mixtures. The solubility of $n$-nonane into liquid water is very small and is therefore neglected. The solubility of methane into the ternary liquids, which are rich in either $n$-nonane or water, is approximated by the solubility of methane into the pure liquid, being either $n$-nonane or water. Likewise, the density of the ternary liquid which is rich in $n$-nonane is approximated by the density of liquid $n$-nonane/methane and the density of the ternary liquid which is rich in water is approximated by the density of

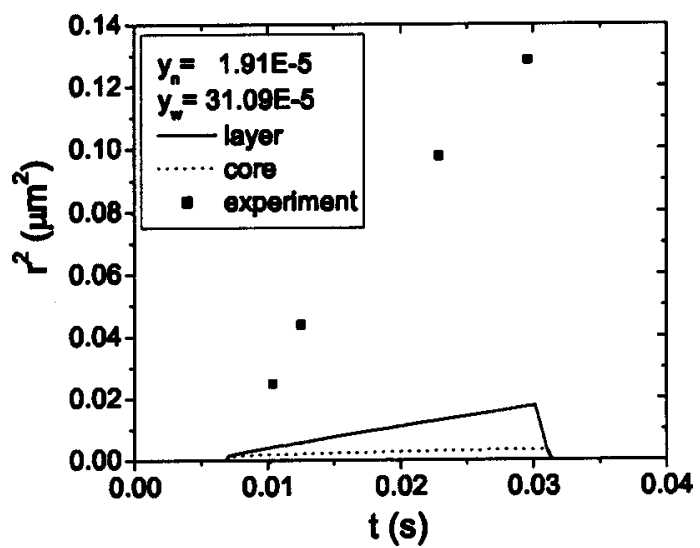

FIG. 10. Growth curve of a water droplet suspended in a mixture of supersaturated water vapor, $n$-nonane vapor, and methane. 


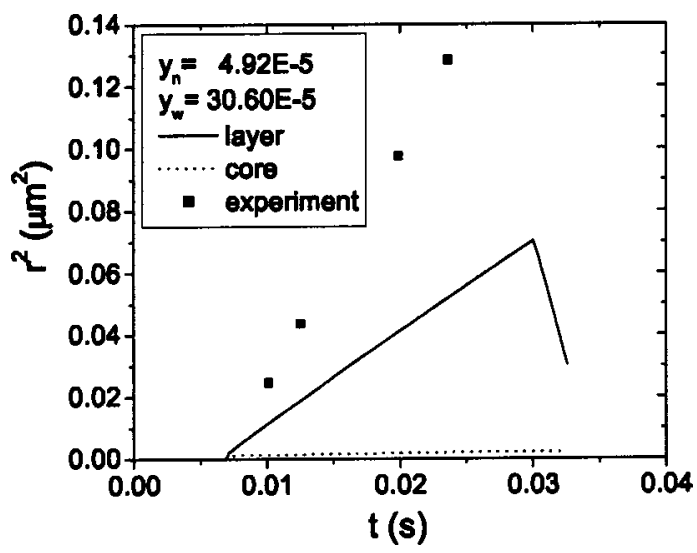

FIG. 11. Growth curve of a water droplet suspended in a mixture of supersaturated water vapor, $n$-nonane vapor, and methane.

liquid water/methane. In Figs. 9-12 four examples of growth curves are shown for the condition of 11 bar and $242 \mathrm{~K}$. In Fig. 9 the $n$-nonane is subsaturated during the growth of the droplet. Hence, it does not form a layer around the water core. For conditions for which the $n$-nonane is supersaturated the model predicts a too small growth rate of the droplets. The difference with the experiments becomes smaller as the vapor fraction of $n$-nonane becomes larger. This holds for all the experiments at 11 bar and $242 \mathrm{~K}$. At 44 bar and $247 \mathrm{~K}$ (not shown) the agreement between the layered droplet calculations and the experiments is somewhat better.

The origin of the deviation for the smaller $n$-nonane vapor fractions becomes more clear when we look at the surface growth rates as a function of the $n$-nonane fraction. The results of the surface growth calculations are shown in Figs. 13 and 14, for the two different conditions studied. The calculations are performed at the fixed averaged conditions (i.e., pressure, temperature, and water vapor fraction) of the relevant experimental series. The droplet number density is fixed at $n_{d}=10^{12} \mathrm{~m}^{-3}$. The surface growth rate is evaluated $5 \mathrm{~ms}$ after the start of the growth, when the depletion of water is still negligible. This roughly corresponds to the appearance of the first Mie peak in the experiments. At this instant the layered model predicts constant surface growth rates for all the experimental conditions considered, as can

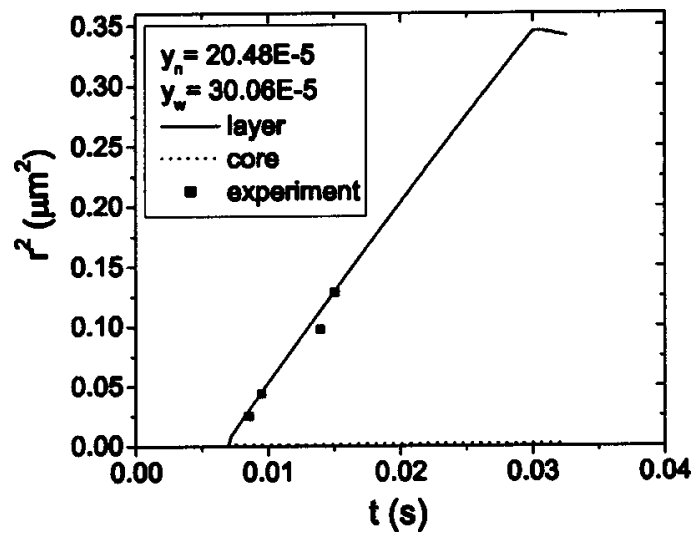

FIG. 12. Growth curve of a water droplet suspended in a mixture of supersaturated water vapor, $n$-nonane vapor, and methane.

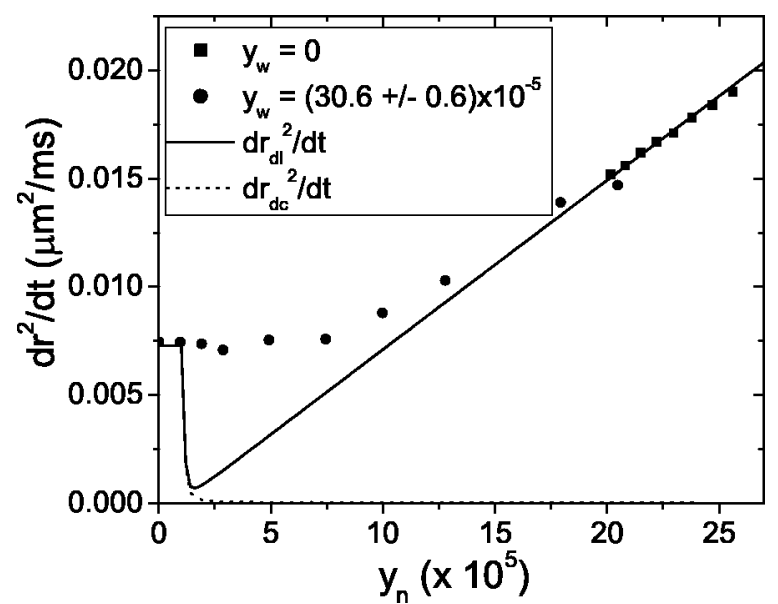

FIG. 13. Surface growth rate of droplets in mixtures of supersaturated $n$-nonane and water in methane as a function of the molar $n$-nonane fraction, at 11 bar and $242 \mathrm{~K}$. Also shown is the surface growth rate of the binary n-nonane/methane mixture.

be seen in the growth plots $\left(r_{d l}^{2}\right.$ versus $\left.t\right)$. The experimental surface growth rates, which are also plotted in Figs. 13 and 14, are time-averaged surface growth rates.

From Fig. 13 it becomes clear that according to the layered droplet model the growth rate of the droplets decreases when the supersaturation of $n$-nonane is small. This can be explained as follows. The volumetric growth rate of the water core is mainly determined by the diffusive flux of water molecules through the $n$-nonane layer. This flux is much smaller than the flux of water molecules through the gas phase towards an uncovered water droplet. Therefore, the volumetric growth rate of the water core is very small compared to the volumetric growth rate of an uncovered water droplet. And, when the supersaturation of the $n$-nonane is small, the volumetric growth rate of the $n$-nonane layer can also be smaller than the volumetric growth rate of the uncovered water droplet, resulting in a smaller overal growth rate for the layered droplet. When the (carrier gas) pressure is increased at constant supersaturation, the ratio of the molar flux of water molecules through the $n$-nonane layer to the

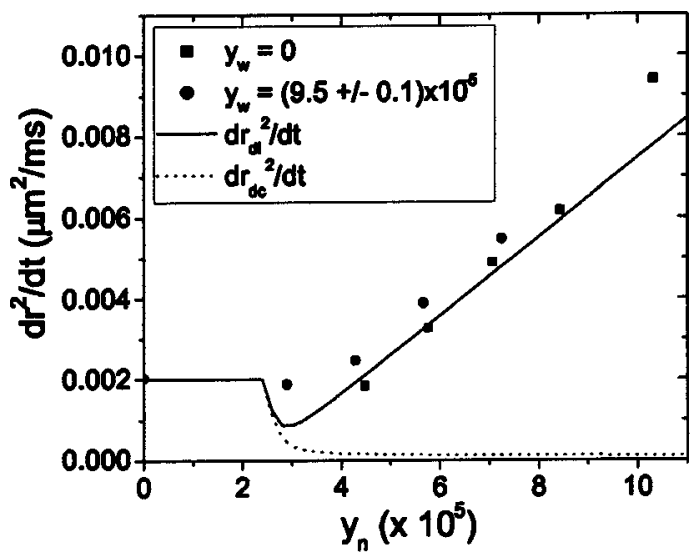

FIG. 14. Surface growth rate of droplets in mixtures of supersaturated $n$-nonane and water in methane as a function of the molar $n$-nonane fraction, at 44 bar and $247 \mathrm{~K}$. Also shown is the (experimentally obtained) surface growth rate of the binary $n$-nonane/methane mixture. 
molar flux of water molecules through the gas phase becomes closer to one, and the effect of the decrease in the growth rate becomes less pronounced. This can be seen in Fig. 14.

The predicted decrease in the droplet growth rates in Fig. 13 is not observed in the experiments. Apparently, in the experiments the water core can grow faster. This cannot be explained by a difference between the actual and calculated value of the diffusion coefficient of water through liquid $n$-nonane. Even increasing this diffusion coefficient by a factor of 10 cannot explain the deviation. Our hypothesis is that the observed difference is caused by $n$-nonane not completely wetting the surface of the water droplet, as is assumed in the layered model. In that case, the $n$-nonane forms one or several caps on the water droplet. The angles between the phases are fixed by the interfacial tensions at the given conditions. Due to cap formation, parts of the water droplet remain uncovered, and the growth of the water droplet is only slightly decreased. As long as the growth rate of the water surface exceeds that of the $n$-nonane cap, the droplet retains its water-rich character and the growth rate is that of a water droplet. If the $n$-nonane cap grows faster than the water surface, it will slowly cover increasingly larger parts of the water surface, until eventually the water droplet is completely covered. Then, the growth can accurately be described by the layered model.

The agreement between the experimentally obtained growth rates in the ternary mixture at 44 bar and $247 \mathrm{~K}$ and the layered growth calculations is better, as can be seen from Fig. 14. Possibly, the complete wetting of $n$-nonane on water is a better approximation at this higher methane pressure. However, the decrease of the growth rate was also expected to become less due to the higher carrier gas pressure.

\section{CONCLUSIONS}

As part of an ongoing research program a new droplet growth model has been constructed. The model is applicable for all Knudsen numbers. It describes the growth of homogeneous multi-component droplets suspended in a gas-vapor mixture. The vapors are diluted in a (real) carrier gas. When compared to the experimental droplet growth results of water and/or $n$-nonane in methane the model performs well. The model can easily be extended to describe the growth of multilayered droplets. Here, this has been done to describe the growth of a droplet consisting of a liquid core and one other outer liquid layer. This model was compared to experimental droplet growth results of supersaturated water and $n$-nonane in methane. The model does not correctly predict the droplet growth results in this case. Most likely, this is caused by the fact that supersaturated $n$-nonane does not form a layer around the water droplet, but rather forms one or more caps on the water droplet. If the caps grow fast enough, they can then eventually form a layer around the water core.

\section{APPENDIX A: ENERGY FLUX IN THE KNUDSEN REGION}

For the determination of the energy flux the gas is first treated as an ideal gas. The properties of the actual gas are then inserted into the final result.

The total molar internal energy of component $i$ in the gas phase is given by

$$
e_{r o t, i}+e_{v i b, i}+e_{\text {tran }, i}=c_{p, i} T-R T \text {. }
$$

When the system is at rest the translational energy is given by

$$
e_{\text {tran }, i}=\frac{3}{2} R T
$$

rendering the following result for the rotational and vibrational energy:

$$
e_{r o t, i}+e_{v i b, i}=\left(c_{p, i}-\frac{5}{2} R\right) T \text {. }
$$

If the translational energy is uncorrelated to the rotational and vibrational energy, the last result also holds when the system is not at rest. The total outgoing energy flux of component $i$ is then given by

$$
\begin{aligned}
\dot{E}_{i}^{+}= & 4 \pi r_{d}^{2} \int_{0}^{\infty} \int_{-\infty}^{\infty} \int_{-\infty}^{\infty} \rho_{d, i} \xi_{r i}\left[\frac{1}{2}\left(\xi_{r i}^{2}+\xi_{\theta i}^{2}+\xi_{\phi i}^{2}\right)+e_{r o t, i}\right. \\
& \left.+e_{v i b, i}\right] f_{i}^{+} \mathrm{d} \xi_{r i} \mathrm{~d} \xi_{\theta i} \mathrm{~d} \xi_{\phi i} \\
= & 4 \pi r_{d}^{2}\left\{\left(e_{r o t, i}+e_{v i b, i}\right) R T_{d} \frac{\rho_{d, i}}{\sqrt{2 \pi M_{i} R T_{d}}}\right. \\
& \left.+2 R^{2} T_{d}^{2} \frac{\rho_{d, i}}{\sqrt{2 \pi M_{i} R T_{d}}}\right\} .
\end{aligned}
$$

Substituting

$$
\dot{M}_{i}^{+}=4 \pi r_{d}^{2} \frac{\rho_{d, i} R T_{d}}{\sqrt{2 \pi M_{i} R T_{d}}},
$$

Eq. (A3) results in

$$
\dot{E}_{i}^{+}=\dot{M}_{i}^{+}\left(c_{p, i}-\frac{R}{2}\right) T_{d}
$$

Of course, $\dot{M}_{i}^{+}=\dot{M}_{i}-\dot{M}_{i}^{-}$, which then gives the first summand of Eq. (22).

If it is again assumed that the translational energy of the molecules is uncorrelated to the rotational and vibrational energy, the total incoming molar translational energy of component $i$ is given by 


$$
\begin{aligned}
\dot{E}_{i}^{-}= & 4 \pi r_{d}^{2} \int_{-\infty}^{0} \int_{-\infty}^{\infty} \int_{-\infty}^{\infty} \frac{1}{2} \rho_{\mathrm{Kn}, i} \xi_{r i}\left(\xi_{r i}^{2}+\xi_{\theta i}^{2}+\xi_{\phi i}^{2}\right) f_{i}^{-} \mathrm{d} \xi_{r i} \mathrm{~d} \xi_{\theta i} \mathrm{~d} \xi_{\phi i} \\
= & \frac{r_{d}^{2}}{5 R T_{\mathrm{Kn}}}\left\{15 \pi M_{i} v_{i} u^{2} \rho_{\mathrm{Kn}, i} R T_{\mathrm{Kn}}+5 \pi M_{i} u^{3} \rho_{\mathrm{Kn}, \mathrm{i}}, R T_{\mathrm{Kn}}+25 \pi u \rho_{\mathrm{Kn}, i} R^{2} T_{\mathrm{Kn}}^{2}+10 \pi \dot{q}_{\mathrm{Kn}, i} R T_{\mathrm{Kn}}\right. \\
& +\left[-15 \pi M_{i} v_{i} u^{2} \rho_{\mathrm{Kn}, i} R T_{\mathrm{Kn}}-5 \pi M_{i} u^{3} \rho_{\mathrm{Kn}, i} R T_{\mathrm{Kn}}-25 \pi u \rho_{\mathrm{Kn}, i} R^{2} T_{\mathrm{Kn}}^{2}-10 \pi \dot{q}_{\mathrm{Kn}, i} R T_{\mathrm{Kn}}\right] \operatorname{erf}\left(\frac{u \sqrt{M_{i}}}{\sqrt{2 \pi R T_{\mathrm{Kn}}}}\right) \\
& +\left[-20 \sqrt{\frac{2 \pi R T_{\mathrm{Kn}}}{M_{i}}} \rho_{\mathrm{Kn}, i} R^{2} T_{\mathrm{Kn}}^{2}+2 \sqrt{2 \pi M_{i} R T_{\mathrm{Kn}}} \dot{q}_{\mathrm{Kn}, i} u-20 \sqrt{2 \pi M_{i} R T_{\mathrm{Kn}}} v_{i} u \rho_{\mathrm{Kn}, i} R T_{\mathrm{Kn}}\right. \\
& \left.\left.-5 \sqrt{2 \pi M_{i} R T_{\mathrm{Kn}}} u^{2} \rho_{\mathrm{Kn}, i} R T_{\mathrm{Kn}}\right] \exp \left(-\frac{u^{2} M_{i}}{2 R T_{\mathrm{Kn}}}\right)\right\} .
\end{aligned}
$$

This exact solution can again be simplified, since $\left|u \sqrt{M_{i}} / \sqrt{2 R T}\right| \ll 1$. First-order Taylor expansion of the exponential and error function then results in

$$
\dot{E}_{i}^{-}=-4 \pi r_{d}^{2}\left[\frac{2 \rho_{\mathrm{Kn}, i} R^{2} T_{\mathrm{Kn}}^{2}}{\sqrt{2 \pi M_{i} R T_{\mathrm{Kn}}}}-\frac{\rho_{\mathrm{Kn}, i} u}{2}\left(\frac{5 R T_{\mathrm{Kn}}}{2}\right)-\frac{\dot{q}_{\mathrm{Kn}, i}}{2}\right] .
$$

The heat flux per unit area is due to conduction and the diffusion of enthalpy,

$$
\dot{q}_{\mathrm{Kn}, i}=\dot{q}_{\mathrm{Kn}, c i}+\rho_{\mathrm{Kn}, i} v_{i}\left(\frac{5 R T_{\mathrm{Kn}}}{2}\right) .
$$

Substituting this into Eq. (A8), together with

$$
\dot{M}_{i}=4 \pi r_{\mathrm{Kn}}^{2} \rho_{\mathrm{Kn}, i}\left(u+v_{i}\right),
$$

then results in

$$
\dot{E}_{i}^{-}=-4 \pi r_{d}^{2} \frac{2 \rho_{\mathrm{Kn}, i} R^{2} T_{\mathrm{Kn}}^{2}}{\sqrt{2 \pi M_{i} R T_{\mathrm{Kn}}}}+\frac{r_{d}^{2}}{2 r_{\mathrm{Kn}}^{2}}\left[\left(\frac{5 R T_{\mathrm{Kn}}}{2}\right) \dot{M}_{i}+\dot{Q}_{c i}\right] \text {, }
$$

where $\dot{Q}_{c i}=4 \pi r_{\mathrm{Kn}}^{2} \dot{q}_{\mathrm{Kn}, c i}$ was used.

\section{APPENDIX B: RADIUS-DEPENDENT FRACTION IN LIQUID LAYER}

The conservation of mass of component $j$ in the liquid layer is given by

$$
\dot{M}_{j}^{L l}-\dot{M}_{j}=\frac{\mathrm{d}}{\mathrm{d} t} \int_{r_{d c}}^{r_{d l} l} x_{j}^{L l}(r) \rho^{L l} 4 \pi r^{2} \mathrm{~d} r .
$$

We assume that the molar density of the liquid layer does not depend on the radius. The molar flux through the liquid layer is given by

$$
\dot{M}_{j}^{L l}=-4 \pi r^{2} \rho^{L l} D_{j}^{L l} \frac{\mathrm{d} x_{j}^{L l}}{\mathrm{~d} r} .
$$

At stationary conditions the mass flux is constant. Therefore, integration of

$$
\int_{x_{j}^{L l}(r)}^{x_{r_{d l}, j}^{L l}} \mathrm{~d} x_{j}^{L l}=\frac{-\dot{M}_{j}^{L l}}{4 \pi \rho^{L l} D_{j}^{L l}} \int_{r}^{r_{d l}} \frac{1}{\left(r^{\prime}\right)^{2}} \mathrm{~d} r^{\prime}
$$

results in the following radius-dependent fraction in the liquid layer:

$$
x_{j}^{L l}(r)=\frac{\dot{M}_{j}^{L l}}{4 \pi \rho^{L l} D_{j}^{L l}} \frac{1}{r}+\left(\frac{-\dot{M}_{j}^{L l}}{4 \pi \rho^{L l} D_{j}^{L l} r_{d l}}+x_{r_{d l}, j}^{L l}\right) .
$$

Inserting this result into the integral of Eq. (B1) yields

$$
\begin{aligned}
\int_{r_{d c}}^{r_{d l}} x_{j}^{L l}(r) \rho^{L l} 4 \pi r^{2} \mathrm{~d} r= & \frac{\dot{M}_{j}^{L l}}{D_{j}^{L l}}\left(\frac{1}{6} r_{d l}^{2}-\frac{1}{2} r_{d c}^{2}+\frac{1}{3} \frac{r_{d c}^{3}}{r_{d l}}\right) \\
& +\frac{4}{3} \pi x_{r_{d l}, j}^{L l} \rho^{L l}\left(r_{d l}^{3}-r_{d c}^{3}\right) .
\end{aligned}
$$

${ }^{1}$ P. Peeters, G. Pieterse, J. Hrubý, and M. E. H. van Dongen, "Multicomponent droplet growth. I. Experiments with supersaturated $n$-nonane vapor and water vapor in methane," Phys. Fluids 16, 2567 (2004).

${ }^{2}$ H. Hertz, "I. ueber die verdunstung der flussigkeiten, insbesondere des quecksilbers, im luftleeren raume," Ann. Phys. (Leipzig) 17, 177 (1882).

${ }^{3}$ M. Knudsen, "Die maximale verdampfungsgeschwindigkeit des quicksilbers," Ann. Phys. (Leipzig) 47, 697 (1915).

${ }^{4} \mathrm{G}$. Gyarmathy, in Multiphase Science and Technology (Springer, Berlin, 1982), Vol. 1, pp. 99-279.

${ }^{5}$ N. A. Fuchs and A. G. Sutugin, Highly Dispersed Aerosols (Ann Arbor Science, Ann Arbor, MI, 1970).

${ }^{6}$ T. Vesala and M. Kulmala, "Comparisons of uncoupled, film theoretical and exact solutions for binary droplet evaporation and condensation," Physica A 192, 107 (1993).

${ }^{7}$ M. Kulmala, T. Vesala, and P. E. Wagner, "An analytical expression for the rate of binary condensational particle growth," Proc. R. Soc. London, Ser. A 441, 589 (1993).

${ }^{8}$ In fact, the expressions given by Gyarmathy result from simplifications of a flux-matching model. The correction factors of Fuchs and Sutugin are obtained from solving the kinetic Boltzmann equations, in which some simplifying assumptions are made.

${ }^{9}$ N. A. Fuchs, "Ueber die verdampfungsgeschwindigkeit kleiner tröpfchen in einer gasatmosphäre," Phys. Z. Sowjetunion 6, 224 (1934).

${ }^{10}$ N. Fukuta and L. A. Walter, "Kinetics of hydrometeor growth from a vapor-spherical model," J. Atmos. Sci. 27, 1160 (1956).

${ }^{11} \mathrm{~J}$. B. Young, "The condensation and evaporation of liquid droplets at arbitrary Knudsen number in the presence of an inert gas," Int. J. Heat Mass Transfer 36, 2941 (1993).

${ }^{12}$ H. Grad, in Encyclopaedia of Physics (Springer, Berlin, 1958), Vol. 12, pp. $205-294$ 
${ }^{13}$ P. Peeters, C. C. M. Luijten, and M. E. H. van Dongen, "Transitional droplet growth and diffusion coefficients," Int. J. Heat Mass Transfer 44, 181 (2001).

${ }^{14}$ C. C. M. Luijten, "Nucleation and droplet growth at high pressure," $\mathrm{Ph} . \mathrm{D}$. thesis, Eindhoven University of Technology, 1998.

${ }^{15}$ I. I. Kolodner, "On the application of the Boltzmann equations to the theory of gas mixtures," Ph.D. thesis, New York University, New York, 1950.

${ }^{16} \mathrm{~J}$. M. Tishkoff, "A model for the effect of droplet interactions on vaporization,” Int. J. Heat Mass Transfer 22, 1407 (1979).

${ }^{17}$ V. V. Sychev, A. A. Vasserman, V. A. Zagoruchenko, A. D. Kozlov, G. A. Spiridonov, and V. A. Tsymarny, Thermodynamic Properties of Methane (Hemisphere, Washington, 1987).

${ }^{18}$ M. J. E. H. Muitjens, "Homogeneous condensation in a vapour/gas mix- ture at high pressures in an expansion cloud chamber," Ph.D. thesis, Eindhoven University of Technology, 1996.

${ }^{19} \mathrm{P}$. Peeters, "Nucleation and condensation in gas-vapor mixtures of alkanes and water," Ph.D. thesis, Eindhoven University of Technology, 2002.

${ }^{20}$ E. C. Voutsas, G. C. Boulougouris, I. G. Economou, and D. P. Tassios, "Water/hydrocarbon phase equilibria using the thermodynamic perturbation theory," Ind. Eng. Chem. Res. 39, 797 (2000).

${ }^{21}$ O. Pfohl, S. Petkov, and G. Brunner, Usage of PE-A Program to Calculate Phase Equilibria (Herbert Utz Verslag, Munchen, 1998), 1st ed.; http://www.tu-harburg.de/vt2/pe2000/HomePage.html.

${ }^{22}$ G. L. Hubbard, V. E. Denny, and A. F. Mills, "Droplet evaporation: effects of transients and variable properties," Int. J. Heat Mass Transfer 18, 1003 (1975). 